\title{
The neuroanatomy of vomiting in man: association of projectile vomiting with a solitary metastasis in the lateral tegmentum of the pons and the middle cerebellar peduncle
}

\author{
PHILIP CH BAKER, JAMES L BERNAT \\ From Napier Hospital, Napier, New Zealand, and Dartmouth Medical School, Hanover, USA
}

SUMMARY Animal studies have indicated a "vomiting center" situated in the dorsal portion of the lateral reticular formation of the medulla at the level of the dorsal nucleus of the vagus. There is also a chemoreceptor trigger zone in the floor of the fourth ventricle in the area postrema which influences the vomiting center. A 63 year old man with a three year history of metastatic malignant melanoma presented with nausea, projectile vomiting, gait ataxia and diplopia associated with horizontal and vertical nystagmus. CT scan showed a solitary brainstem metastasis without hydrocephalus and he was treated with radiotherapy with resolution of his vomiting after four weeks. At post mortem three months later a metastasis was found in the right middle cerebellar peduncle and lateral tegmentum of the pons; there was no pathological change in the area of the vomiting center or area postrema. It is postulated that this lesion caused projectile vomiting because of involvement of either afferent or efferent projections to the vomiting center. The neuroanatomy of vomiting is discussed.

Clinical studies have shown that there is a "vomiting centre" situated in the dorso-lateral reticular formation of the medulla with a chemoreceptor trigger zone for vomiting in the area postrema in the floor of the caudal part of the fourth ventricle.' The vomiting centre was defined by Borison and Wang in $1949^{2}$ in a series of experiments on decerebrate cats. They showed that electrical stimulation of the lateral reticular formation in the vicinity of the fasciculus solitarius, but no other region of the brain-stem, resulted in immediate projectile vomiting which continued for as long as the stimulus was applied. Hess $^{3}$ found that stimulation of points in the diencephalon and forebrain occasionally produced vomiting but this consisted of rhythmic retching movements rather than projectile vomiting and generally there was some delay in onset. The vomiting centre

Address for reprint requests: PCH Baker, Napier, New Zealand.

Received 11 December 1984 and in revised form 26 April 1985. Accepted 10 May 1985 in the medullary reticular formation is not itself sensitive to centrally-acting emetic drugs such as apomorphine but rather these drugs act on a separate receptor area which, in the cat, is located in the area postrema; this area differs from surrounding brain tissue in that it is permeable to many particulate substances such as tryphan blue, indicating a breakdown in the "blood-brain barrier." Ablation of the area postrema results in abolition of the emetic response to intravenous apomorphine whereas the response to oral copper sulphate (which is presumably mediated through afferent nerve fibres from the gut), is not affected unless the ablation also involves the lateral reticular formation.

Projectile vomiting in man has been associated with conditions of raised intracranial pressure, and, more particularly, with lesions of the posterior fossa. The pathophysiology of this response however has not been clearly defined. The patient who is reported in this paper is of interest in that he presented with projectile vomiting and was found to have a single lesion, which did not directly involve 
the medullary reticular formation or the caudal part of the fourth ventricle.

\section{Case report}

A 63-year old-man had a malignant melanoma removed

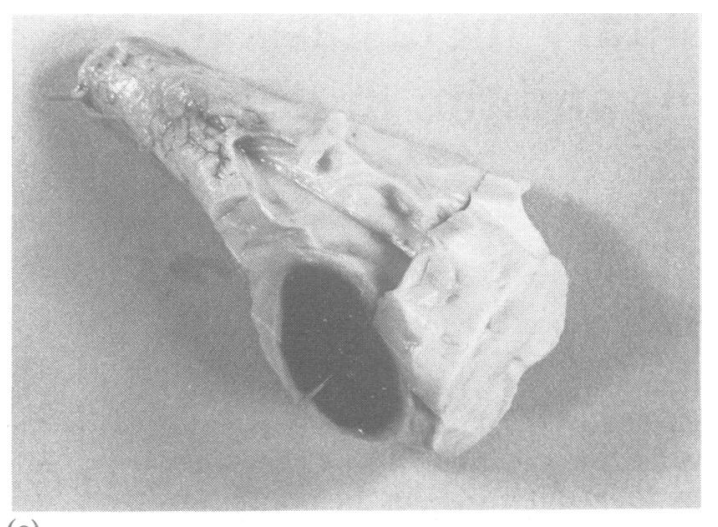

(a) from his back three years earlier; he subsequently had a single lung metastasis removed and later developed metastases in the bowel and buttock. Over the course of several days he developed nausea, projectile vomiting, diplopia and ataxia. Lying down flat or leaning forwards produced vertigo and vertical diplopia, together with sudden, uncontrollable forceful vomiting; when he sat or stood still however he was reasonably comfortable. He had marked horizontal nystagmus on looking to either side, more on the right than the left, and vertical nystagmus on looking upwards. There was no apparent paresis of eye movement but looking upwards and laterally to either side produced vertical diplopia, with the abnormal image coming from the abducted eye. Pupils reacted normally and there were normal corneal reflexes, facial sensation, facial movement, hearing, gag reflex, speech and tongue movement. Motor and sensory examination, deep tendon reflexes and plantar responses were all normal. Tests of fine hand movements showed slight dysmetria bilaterally but his gait was markedly ataxic with falling to either side. A CT scan showed a single posterior fossa lesion extending from the lateral margin of the pons into the right cerebellum; all four ventricles were normal in size. He was treated with high-dose steroids and 2800 whole-brain irradiation; he also had radiotherapy for recurrent metastases in the chest and buttock. Vomiting improved after four weeks but the gait

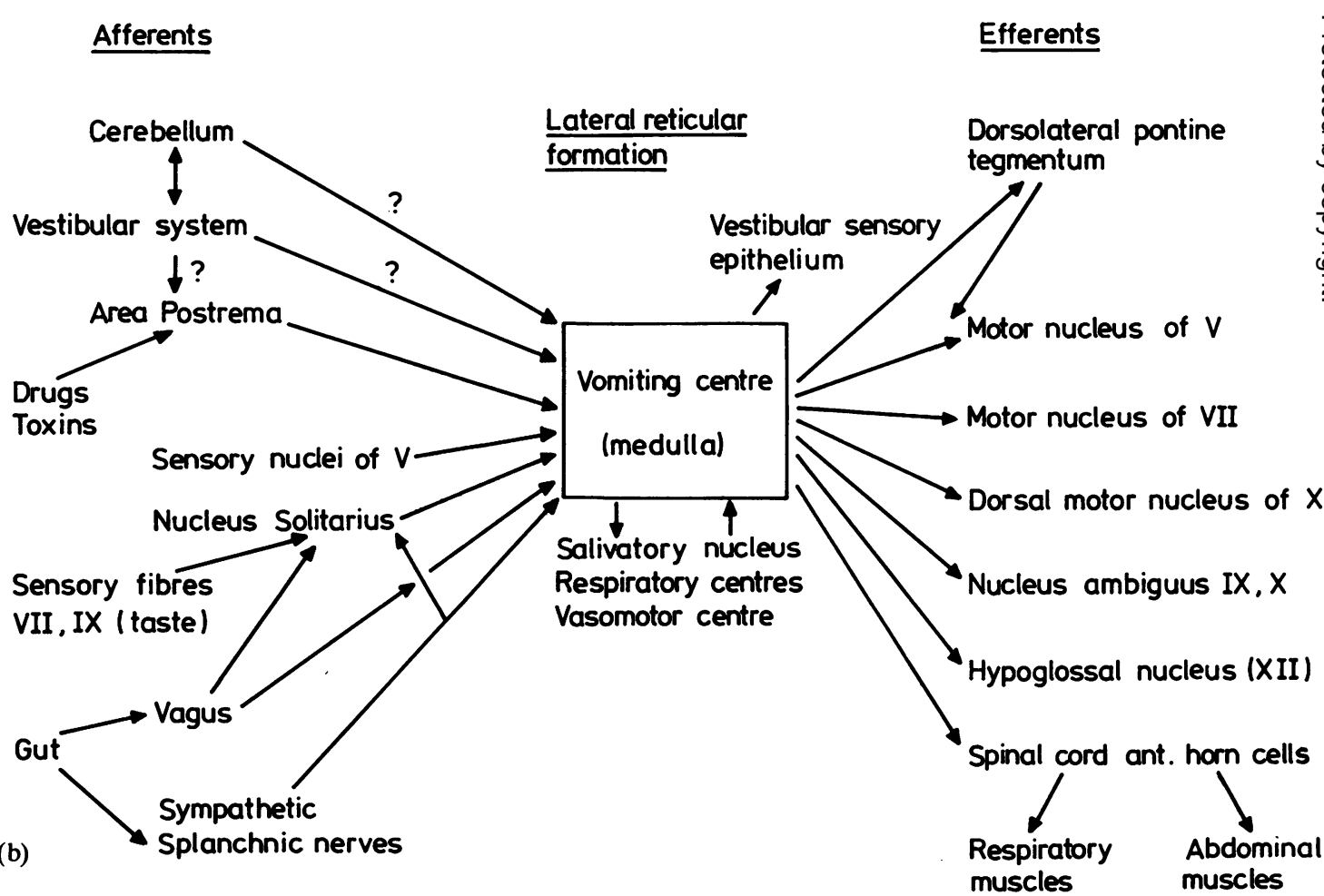

Fig (a) Dorsal view of the brain-stem showing malignant melanoma centred on the right middle cerebellar peduncle. Tumour also extends into the lateral tegmentum of the pons. (b) Major afferent and efferent pathways to the vomiting centre. ? indicates that the assumed pathway has not been identified. 
ataxia did not change. He later developed pneumonia and septicaemia and died about three months after his initial neurological presentation.

\section{Pathology}

At necropsy a solitary brain metastasis was found (fig, a); there was no hydrocephalus or any other pathological change in the rest of the brain. The metastasis was centred on the right middle cerebellar peduncle and also involved a portion of the lateral basis pontis and the lateral tegmentum of the pons. Its caudal extent was marked by the facial colliculus and rostrally it reached no further than the isthmus; laterally it extended into the corpus medullare but did not involve cerebellar cortex. In its caudal portion it included the superior and lateral vestibular nuclei and therefore abutted the pontine lateral reticular formation; however it did not extend into the medulla at all. The tumour was close to but did not involve the floor of the rostral fourth ventricle and it did not obstruct or distort the anatomy of the ventricle.

\section{Discussion}

This patient had a single lesion which did not involve either the classical vomiting centre in the medullary lateral reticular formation or the chemoreceptor trigger zone. We have considered two main possibilities to account for his symptom of projectile vomiting. Firstly, he probably had raised intracranial pressure and movements of his head may have caused further transient rises in pressure resulting in vomiting. Against this suggestion is the fact that he did not have hydrocephalus and the location and size of the lesion, both on CT and post mortem, suggest that intermittent hydrocephalus would be unlikely to occur. If raised intracranial pressure is the cause of projectile vomiting the question can still be asked what part of the vomiting mechanism is affected by this factor; raised pressure in the IVth ventricle could possibly exert an effect on the area postrema, but we are not aware of any experimental evidence to support this. Certainly any theory would have to explain why projectile vomiting is much more common with lesions in the posterior fossa than with metabolic encephalopathies which cause generalised cerebral oedema. Secondly, the lesion may involve either efferent or afferent connections to the vomiting centre. Major efferent pathways are found between the lateral reticular formation (LRF) and the cranial nerve motor nuclei controlling jaw, mouth and tongue movements and there are also pathways to the motor neurons that control respiratory muscles and the striated abdominal muscles that are of prime importance in the expulsive phase of vomiting. Both efferent and afferent connections are made between the vomiting centre and the various other centres in or adjacent to the LRF; these include the inspiratory and expiratory respiratory centres; the vasomotor centre and the salivatory nucleus, each of which represents activities that form part of the motor expression of vomiting. The principal afferents to the vomiting centre come from the area postrema and from the gut via both vagus and sympathetic splanchnic nerves, often with a relay in the adjacent nucleus solitarius. None of these pathways helps to explain the cause of vomiting in our patient.

Since motion is a common cause of nausea and vomiting it has long been assumed that there must be some connection between the vestibular system and the LRF. Labyrinthectomy, or section of the eighth cranial nerves, or removal of the cerebellar nodulus and pyramis prevents motion-induced vomiting in animals. ${ }^{4}$ Removal of the cerebellum does not however reduce the emetic action of drugs such as apomorphine, whereas if animals are made insensitive to the action of apomorphine by ablation of the area postrema, they are also rendered insensitive to the emetic action of motion. ${ }^{6}$ This suggests that the area postrema is in the direct pathway between the vestibular system and the vomiting centre. However, to date this pathway has not been defined and no direct vestibular-reticular or cerebello-reticular pathways have been found, with the exception of the cerebello-reticular fibres found by Ramon y Cajal in $1903,{ }^{7}$ which have only been identified in the rat and guinea-pig and not in other species.

More recently it has been shown that there are efferent fibres to the vestibular sensory epithelium of the semi-circular cells which have their origin partly in the vestibular nuclei, but mainly in the LRF at the level of the upper medulla and lower pons; ${ }^{8}$ this part of the LRF in its rostral extent is close to the tumour found in our patient. It has been speculated that the vestibular efferents modulate the dynamic range of vestibular afferents to match expected acceleration of the head, and it is possible that mis-matching of vestibular impulses could contribute to nausea and/or vomiting. Motion sickness however differs from projectile vomiting in that nausea and retching are prominent and therefore the same connection between the vestibular system and the vomiting centre would not be expected to subserve both processes.

Recent studies using the technique of horse radish peroxidase injections have shown that there are many more connections between the LRF (now also termed the parvicellular reticular formation) and other brain-stem structures (fig, b). This subject has recently been reviewed by Mehler. ${ }^{9}$ Of particular interest is the finding of ascending fibres from the LRF of the medulla and from the area postrema to the dorso-lateral pontine tegmentum where they terminate on or ventral to the parabrachial nuc- 
lei. ${ }^{9-11}$ There are also descending projections from the dorso-lateral tegmentum of the pons (from the Koelliker-Fuse nucleus which is ventro-lateral to the parabrachial nuclei), to the facial, ambiguus and hypoglossal nuclei and motor neurons of the spinal cord. ${ }^{12}$ Since the lesion in our patient was located in this same area of the tegmentum of the pons it is possible that involvement of these pathways may have been the cause of his projectile vomiting.

Finally, it is interesting to turn to other case reports. Adams and Victor in their textbook Principles of Neurology, state that the most persistent vomiting they have seen is in patients who have a posterior fossa lesion and they cite a case of a brain-stem glioma and a posterior fossa meningioma without otherwise specifying the location of the lesions. The most useful information comes from Lindstrum and Brizzee who report five patients with intractable vomiting, whom they treated by bilateral ablation of the area postrema. ${ }^{13}$ In each case spontaneous vomiting and apomorphine-sensitive vomiting were abolished and this was achieved without producing any other neurological sequelae. This report is important in confirming that the chemoreceptor trigger zone in man is located in the area postrema and also establishes a safe form of treatment for intractable vomiting. It also provides further information about the nature of the lesions that can produce severe vomiting. In two of their cases there appeared to be a generalised cerebral dysfunction; the diagnosis in one was a non-specific encephalitis principally affecting brain-stem function and with features of raised intracranial pressure; in the second, self-induced vomiting following a possible encephalitis. The third patient was assumed to have a brain-stem glioma; she had occipital pain, diplopia, ataxia, dysarthria and raised intracranial pressure with papilloedema, as well as vomiting, but the lesion was not found at the time of operation for thermal ablation of the area postrema. The two final patients had clearly defined lesions in the posterior fossa; one had a single metastatic lesion in the right cerebellum and the other had metastases in the midbrain, including "the posterior half of the third ventricle and the left superior half of the fourth ventricle" (therefore including the dorso-lateral tegmentum of the pons). It is interesting to note that both the third and fifth patients were exquisitely sensitive to movement; it is commented that they lay "motionless" in bed since even slight movements induced vomiting". This sensitivity to movement, which was also seen in our patient, again raises the possibility of vestibular connections being involved in the genesis of their vomiting.

In conclusion, while it is known that there is a vomiting centre in the medulla, there is still much to be learnt about the afferent and efferent connections, and the exact pathophysiology of the various types of vomiting has yet to be defined. The location of a lesion causing projectile vomiting in man cannot be predicted reliably but the clinico-pathological evidence we have presented suggests that there is more likely to be involvement of the dorso-lateral tegmentum of the pons or the adjacent cerebellar peduncles rather than the medulla.

\section{References}

${ }^{1}$ Borison HL, Wang SC. Physiology and pharmacology of vomiting. Pharmacol Rev 1953;5:193-230.

${ }^{2}$ Borison HL, Wang SC. Functional localization of central co-ordinating mechanism for emesis in cat. $J$ Neurophysiol 1949;12:305-13.

${ }^{3}$ Hess WR. Vegetative Funktionen und Zwischenkirn. Helvet Physiol Acta 1947; Suppl 4.

${ }^{4}$ Bard P, Woolsey CN, Snider RS, et al. Determination of central nervous mechanisms involved in motion sickness. Fed Proc 1947; 6: 72.

5 Tyler DB, Bard P. Motion sickness. Physiol Rev 1949;29:311-69.

- Wang SC, Chinn HI. Emetic trigger zone and motion sickness in dogs. Fed Proc 1952;11:400.

${ }^{7}$ Ramon y Cajal. La doble via descendente nacida del pedunculo cerebelloso superior. Trab Lab Invest Biol Univ Madrid 1903;2:23-29.

${ }^{8}$ Ito J, Takahashi H, Matsuoka I et al. Vestibular efferent fibres to ampulla of anterior, lateral and posterior semi-circular canals in cats. Brain Res 1983; 259:293-7.

${ }^{9}$ Mehler WR. Observations on the connectivity of the parvicellular reticular formation with respect to a vomiting center. Brain Behav Evol 1983;23:63-80.

${ }^{10} \mathrm{King} \mathrm{GW}$. Topology of ascending brainstem projections to nucleus parabrachialis in the cat. J Comp Neurol 1980;191:615-38.

"Shapiro RE, Miselis RR. An efferent projection from the area postrema and the caudal medial nucleus of the solitary tract to the parabrachial nucleus in rat. Neurosci Abstr 1982;8:269.

${ }^{12}$ Mizuno N, Nomura S, Takeuchi Y. The parabrachial nucleus as an intermediate relay station of the visceral afferent pathways in the cat, In Ito: Integrative Control Function of the Brain. Vol. 3. Amsterdam, Elsevier, 1981. pp 1-20.

${ }^{13}$ Lindstrom PA, Brizzee KR. Relief of intractable vomiting from surgical lesions in the area postrema. $J$ Neurosurg 1962;228-36. 